\title{
Caracterização morfológica e química da madeira de Schizolobium amazonicum aos 14 anos de plantios no estado de Rondônia
}

\author{
Morphological and chemical characterization of the wood of \\ Schizolobium amazonicum at 14 years of plantations in the state of Rondônia
}

\author{
Karina Soares Modes', Geraldo Bortoletto Júnior ${ }^{2}$, Mario Tomazello Filho ${ }^{3}$, \\ Lourdes Maria Hilgert Santos ${ }^{4}$, Magnos Alan Vivian ${ }^{5}$ e Francides Gomes da Silva Júnior ${ }^{2}$
}

\begin{abstract}
Resumo
O presente estudo teve o objetivo de verificar a variação radial das características de fibras e vasos e a composição química da madeira de paricá (Schizolobium amazonicum) aos 14 anos, proveniente de duas áreas de florestas plantadas no estado de Rondônia. Foram amostradas 4 árvores de cada plantio e destas removidos discos a $0,60 \mathrm{~m}$ do nível do solo para caracterização anatômica de fibras e vasos nas posições $0 \%, 25 \%, 50 \%, 75 \%$ e $100 \%$ do comprimento do raio, e discos nesta mesma posição e em outras duas, distantes $2,0 \mathrm{~m}$ para a caracterização da composição química da madeira. As médias de comprimento de fibras, espessura de parede, largura de fibras e diâmetro de lume foram respectivamente $1,30 \mathrm{~mm}$, $2,80 \mu \mathrm{m}, 31,10 \mu \mathrm{m}$ e $25,50 \mu \mathrm{m}$, sendo que tanto o comprimento como a espessura de parede mostrou estabilização na posição a $75 \%$ do raio, para a madeira de ambos os plantios avaliados. Os vasos apresentaram um diâmetro médio de 194,08 um, com uma área ocupada de 5,28\% e frequência de 1,83 vasos/ $\mathrm{mm}^{2}$. A composição química média da madeira indicou uma porcentagem de extrativos de $6,65 \%$, teor de holocelulose de $64,71 \%$, lignina total de $28,63 \%$ e teor de cinzas de $0,61 \%$. Os parâmetros referentes às fibras não foram influenciados pela proveniência da madeira e tomando-se por base a variação radial dos parâmetros de comprimento e espessura de parede das fibras, já se observa a presença de madeira adulta nesta espécie aos 14 anos nas condições de crescimento avaliadas. A madeira dos plantios avaliados diferenciou-se quanto ao diâmetro de vaso e sua área ocupada, bem como em relação ao teor de cinzas.
\end{abstract}

Palavras-chave: Paricá; Fibras; Vasos; Holocelulose

\begin{abstract}
This study aimed to determine the radial variation of the fiber characteristics and vessels and the chemical composition of parica wood (Schizolobium amazonicum) at age 14 from two planted forests in Rondônia. Four trees were sampled from each plantation and discs removed at $0.60 \mathrm{~m}$ up from soil level for anatomical characterization of fibers and vessels at $0 \%, 25 \%, 50 \%, 75 \%$ and $100 \%$ in the pith-to-bark direction; from discs in this same position and in another two, distant $2.0 \mathrm{~m}$ for the characterization of the chemical composition of the wood. The mean fiber length, thickness, width and lumen diameter were respectively $1.30 \mathrm{~mm}, 2.80 \mu \mathrm{m}, 31.10 \mu \mathrm{m}$ and $25.50 \mu \mathrm{m}$; both the fiber length and thickness showed stabilization at $75 \%$ of the pith-to-bark direction for the wood of both evaluated plantations. The vessels had a mean diameter of $194.08 \mu \mathrm{m}$, an occupied area and frequency of $5.28 \%$ and 1.83 vessels $/ \mathrm{mm}^{2}$, respectively. The mean chemical composition of the wood indicated a percentage of extractives of $6.65 \%$; holocellulose content of $64.71 \% ; 28.63 \%$ of the total lignin and $0.61 \%$ of ash content. The parameters related to the fibers were not influenced by the origin of the wood. Based on the radial variation of the parameters of length and wall thickness of the fibers, the presence of mature wood in this species at age 14 was observed under the

1 Professora Doutora do Departamento de Agricultura, Biodiversidade e Florestas. UFSC - Universidade Federal de Santa Catarina / Centro de Ciências Rurais. Rodovia Ulysses Gaboardi, km 3 - 89.520-000, Curitibanos-SC. E-mail: karina.modes@ufsc.br.

2Professor Associado do Departamento de Ciências Florestais. USP - Universidade de São Paulo / ESALQ - Escola Superior de Agricultura Luiz de Queiroz. Av. Padua Dias, 11 - Caixa Postal: 9 - 13418-900 - Piracicaba, SP, Brasil. E-mail: gbjunior@usp.br; fjr@usp.br.

3Professor Titular do Departamento de Ciências Florestais. USP - Universidade de São Paulo / ESALQ - Escola Superior de Agricultura Luiz de Queiroz. Av. Padua Dias, 11 - Caixa Postal: 9 - 13418-900 - Piracicaba, SP, Brasil. E-mail: mtomazel@usp.br.

${ }^{4}$ Mestranda em Ciências Florestais. UFES - Universidade Federal do Espírito Santo / Centro de Ciências Agrárias e Engenharias. Avenida Governador Lindemberg, 316 - 29550000 - Jerônimo Monteiro, ES, Brasil. E-mail: lourdesmaria.engflorestal@yahoo.com.br.

${ }^{5}$ Professor Doutor de Departamento de Agricultura, Biodiversidade e Florestas. UFSC - Universidade Federal de Santa Catarina, Campus Curitibanos. Rod. Ulysses Gaboardi - km 3 - 89520000 - Curitibanos, SC, Brasil. E-mail: magnosalan@gmail.com.
\end{abstract}


evaluated growing conditions. The wood of the evaluated plantations differed in the diameter of vessels and its occupied area as well as with respect to ash content.

Keywords: Paricá; Fibers; Vessels; Holocellulose

\section{INTRODUÇÃO}

Segundo Iwakiri et al. (2010), a implantação de florestas com espécies nativas na Região Norte foi impulsionada pela preocupação do setor florestal e madeireiro com a imagem negativa da devastação da floresta amazônica e pelas pressões ambientalistas sobre o uso industrial de madeiras tropicais sem manejo florestal sustentado.

Conforme Lobão (2011) há grande demanda por madeira de árvores de espécies tropicais, tal como a de Schizolobium parahyba var. amazonicum (sinonímia empregada por alguns autores para designar a espécie em referência), porém, é escassa a literatura sobre a qualidade do seu lenho. De acordo com Terezo (2010), com uma produtividade que varia de 13 a $35 \mathrm{~m}^{3} / \mathrm{ha} /$ ano, a espécie Schizolobium amazonicum considerada de rápido crescimento tornou-se, em meados da década de 1990, uma alternativa viável para a implantação de novas florestas plantadas na Região Norte e Centro-Oeste do Brasil.

Para Jesus (2004) a variação das dimensões dos componentes anatômicos do lenho tem um efeito marcante sobre a qualidade da madeira e constitui-se no elemento fundamental na análise das alternativas de possíveis utilizações que se pretenda dar a este material. Adicionalmente, o conhecimento de tal variação também é importante para investigar as modificações da madeira ocasionadas por diferenças ambientais. Para Vidaurre (2010) o estudo da qualidade da madeira permite uma melhor adequação da matéria-prima a um determinado uso, ou a sua capacidade para preencher os requisitos necessários à fabricação de um determinado produto.

A caracterização química e anatômica da madeira da espécie Schizolobium amazonicum no estado de Rondônia, o qual se encontra entre aqueles de ocorrência natural da referida espécie no País, permitirá dar contribuição à investigação científica que já vem sendo conduzida em outros estados, bem como prever o grau de variabilidade existente para os parâmetros anatômicos e químicos encontrados com os já relatados na literatura, com vistas a confirmar, e/ou ampliar, as possibilidades de uso até hoje previstas para a sua madeira, a partir de indivíduos em idade mais avançada. Também, a partir da análise de propriedades químicas e anatômicas da madeira de Schizolobium amazonicum de plantios distintos, pretende-se verificar o grau de alteração existente, em resposta a características de solo de reconhecida influencia, de acordo com informações disponíveis na literatura, possibilitando oferecer a base para estudos futuros com relação a esta temática.

Diante do exposto, o presente estudo foi conduzido com o objetivo de avaliar a variação radial das características de fibras e vasos e a composição química da madeira de Schizolobium amazonicum aos 14 anos, proveniente de duas áreas distintas de florestas plantadas no estado de Rondônia.

\section{MATERIAL E MÉTODOS}

\section{Caracterização dos plantios, coleta e preparo do material}

Para o estudo foram amostradas árvores da espécie Schizolobium amazonicum Huber ex Ducke com 14 anos de idade, proveniente de duas diferentes áreas de cultivo no estado de Rondônia, localizadas nos municípios de Rolim de Moura ( $11^{\circ} 48^{\prime}$ de latitude sul; $61^{\circ} 48^{\prime}$ de longitude oeste e $271 \mathrm{~m}$ de altitude) e de Parecis $\left(12^{\circ} 07^{\prime}\right.$ de latitude sul; $61^{\circ} 36^{\prime}$ de longitude oeste e $374 \mathrm{~m}$ de altitude). Segundo a classificação de Köppen, o estado de Rondônia possui um clima do tipo Aw (Clima Tropical Chuvoso), com estação seca definida entre os meses de maio a outubro e chuvosa entre novembro a abril.

No total foram amostradas oito árvores, sendo quatro para cada uma das referidas áreas, cujos plantios foram estabelecidos no espaçamento 2,0 x 2,0 metros. Os valores médios de Diâmetro à Altura do Peito (DAP) e altura comercial das árvores amostradas foram 0,44 m e 16,7 m, respectivamente. O corte dos indivíduos amostrados ocorreu na altura de 0,60 m do nível do solo e nesta posição foi amostrado um disco de 3,0 cm de espessura para os procedimentos de caracterização 
anatômica da madeira e outro de $2,0 \mathrm{~cm}$ de espessura para a caracterização da composição química. À distância de 2,0 e 4,0 m desta posição foram amostrados outros dois discos ainda para a caracterização química.

Para caracterização das áreas de ocorrência dos plantios foi conduzida a amostragem de solo, segundo metodologia adaptada de Castelo et al. (2008), para estudo dos atributos físico-químicos. Para isso foi realizada amostragem de solo a granel a uma distância de $1 \mathrm{~m}$ dos indivíduos amostrados, em dois pontos distintos, nas profundidades 0 a $10 \mathrm{~cm} \mathrm{e} 10$ a $30 \mathrm{~cm}$, gerando uma amostra composta para análise química. Também, em dois pontos distintos, nas posições 10 e $40 \mathrm{~cm}$ de profundidade foram coletadas amostras indeformadas (através de anéis metálicos), para estudo dos atributos físicos.

A determinação dos atributos físicos do solo foi conduzida no Laboratório de Análises Físicas do Solo da Universidade Federal de Santa Maria (UFSM). Já os atributos químicos foram determinados no Laboratório de Solos da Escola Superior de Agricultura "Luiz de Queiroz" (ESALQ/USP).

\section{Morfologia de vasos e fibras}

Para este procedimento, a partir dos discos amostrados, foram confeccionadas baguetas, passando pela medula, e destas retirados blocos, de seção quadrada 1,5 a 2,0 cm de posições correspondentes a 0, 25, 50, 75 e 100\% do comprimento do raio dos discos. Para obtenção de informações acerca do diâmetro $(\mu \mathrm{m})$, frequência (vasos $/ \mathrm{mm}^{2}$ ) e área ocupada por vasos (\%), foi preparada uma lâmina histológica temporária com auxílio de micrótomo de deslize do plano transversal de cada posição radial.

Para determinação das dimensões das fibras tais como comprimento $(\mathrm{mm})$, largura $(\mu \mathrm{m})$, espessura da parede celular $(\mu \mathrm{m})$, e diâmetro do lume $(\mu \mathrm{m})$, um fragmento da aresta no sentido das fibras dos mesmos corpos-de-prova empregados na caracterização histológica da madeira, foi submetido à técnica de maceração pelo Método Franklin. A mensuração dos parâmetros descritos, em relação às células de vaso e fibra, se deu por meio do software Image Pro Plus, baseada nas normas da International Association of Wood Anatomists (IAWA COMMITTEE, 1989).

Adicionalmente a partir dos parâmetros morfológicos das fibras foram calculados os coeficientes indicativos de qualidade da madeira de $S$. amazonicum para produção de polpa e papel, tais como fração parede (\%), coeficiente de flexibilidade (\%) e índice de Runkel, segundo metodologia de Foelkel e Barrichelo (1975).

\section{Caracterização química da madeira}

Para a caracterização química da madeira foi confeccionada uma cunha de cada um dos discos com 2,0 cm de espessura, cortados ao longo de três posições longitudinais de cada árvore. As cunhas obtidas foram transformadas em cavacos, reduzidos a serragem em moinho de facas e após o peneiramento selecionadas a fração de serragem que passou pela peneira de 40 mesh $(0,42 \mathrm{~mm})$ e ficou retida na peneira de 60 mesh $(0,25 \mathrm{~mm})$, que foi misturada gerando uma amostra composta por árvore. Posteriormente, essas amostras foram submetidas à análise para determinação da sua composição química, conduzida no Laboratório de Química, Celulose e Energia (LQCE) da ESALQ/USP. Foram determinados os teores de extrativos e de lignina, considerando a metodologia preconizada pela TAPPI T 204 cm-97 (TAPPI, 2007) e TAPPI T 222 om-02 (TAPPI, 2007). O teor de holocelulose foi determinado considerando a soma dos teores de extrativos e lignina total. Já o teor de cinzas foi determinado conforme recomendação da norma TAPPI T 211 om-02 (TAPPI, 2007).

\section{Análise estatística}

Os parâmetros anatômicos foram avaliados a partir de delineamento inteiramente casualizado com arranjo bifatorial, sendo os fatores: posição radial com 5 níveis (0\%, 25\%, 50\%, 75\% e 100\%) e proveniência com 2 níveis (Parecis e Rolim de Moura), totalizando 10 tratamentos, com 4 repetições cada. Foi verificada a normalidade dos dados (teste Shapiro-Wilk, a 5\% de significância), para posterior análise de variância (ANOVA) e comparação de médias pelo teste de Tukey, em nível de $1 \%$ e $5 \%$ de probabilidade de erro, sendo que para avaliação da composição química da madeira foi empregada apenas esta última. Foi utilizada a correlação linear simples (Pearson) para verificar a correlação entre atributos químicos e físicos do solo e as propriedades químicas e anatômicas da 
Modes et al. - Caracterização morfológica e química da madeira de

Schizolobium amazonicum aos 14 anos de plantios no estado de Rondônia

madeira, aplicando-se o programa estatístico ASSISTAT Versão 7.7.

\section{RESULTADOS E DISCUSSÃO}

\section{Caracterização morfológica \\ Morfologia de fibras da madeira de Schizolobium amazonicum}

Observa-se que os parâmetros referentes às fibras da madeira de S. amazonicum não foram influenciados pela proveniência dos indivíduos amostrados (tabela 1).

Tabela 1. Teste de médias para as variáveis de fibra da madeira de S. amazonicum aos 14 anos em função da proveniência.

Table 1. Mean test for S. amazonicum wood fiber at age 14, by origin.

\begin{tabular}{lcccc}
\hline \multirow{2}{*}{ Proveniência } & \multicolumn{4}{c}{ Dimensões das Fibras } \\
\cline { 2 - 5 } & $\begin{array}{c}\text { Comprimento } \\
(\mathbf{m m})\end{array}$ & $\begin{array}{c}\text { Espessura de } \\
\text { parede }(\boldsymbol{\mu m})\end{array}$ & $\begin{array}{c}\text { Largura de } \\
\text { fibras }(\boldsymbol{\mu m})\end{array}$ & $\begin{array}{c}\text { Diâmetro de } \\
\text { lume }(\boldsymbol{\mu m})\end{array}$ \\
\hline Parecis & $1,30 a^{*}$ & $2,89 a$ & $30,94 a$ & $25,16 a$ \\
Rolim de Moura & $1,31 \mathrm{a}$ & $2,71 \mathrm{a}$ & $31,25 \mathrm{a}$ & $25,83 a$ \\
Média & 1,30 & 2,80 & 31,10 & 25,50 \\
$\mathrm{CV}(\%)$ & 7 & 14 & 11 & 16 \\
\hline Em que: * Médias seguidas pela mesma letra não diferem estatisticamente entre si pelo teste de Tukey $(\mathrm{p} \geq 0,05) ; \mathrm{CV}=$ Coeficiente de variação
\end{tabular}

Quanto ao comprimento de fibras, o valor médio do presente estudo é superior em $23,84 \%$ ao verificado por Urbinati (2013) em indivíduos de mesma idade porém equivalente aquele registrado por Terezo (2010) na idade de 19 anos.

Quanto a espessura de parede, o valor médio registrado $(2,80 \mu \mathrm{m})$ é compatível ao registrado nos estudos de Ribeiro (2015) e Lobão et al. (2012) a partir de árvores com idades entre 10 e 18 anos.

No que se refere ao diâmetro de lume das fibras, o valor médio é próximo ao encontrado em um povoamento de 11 anos estudado por Vidaurre (2010). Quanto à largura de fibras, o valor registrado é compatível com o verificado por Lobão et al. (2012) a partir de indivíduos em idade mais avançada (22 a 33 anos).

Silva et al. (2016) observaram em estudo anatômico com a madeira de Schizolobium parahyba var. amazonicum procedente de três municípios do estado do Pará, diferenças significativas quanto às dimensões das fibras e atribuíram o fato as diferenças climáticas entre os municípios, influenciando a disponibilidade hídrica, que por sua vez condiciona a expansão e divisões celulares, bem como as potencialidades genéticas e características fenotípicas. Da mesma forma Lobão et al. (2012) também verificaram diferenças anatômicas entre fibras da madeira de Schizolobium parahyba var. amazonicum de duas localidades distintas no estado do Acre.

Considerando, por sua vez, que a literatura relata diferenças entre parâmetros anatômicos para a espécie $S$. amazonicum em crescimento em localidades inseridas dentro de um mesmo estado, é de se esperar, no presente estudo, resultados divergentes com a literatura, que podem estar relacionados às condições climáticas reinantes no estado de Rondônia, qualidade do sítio de crescimento, bem como em relação à procedência genética da semente.

A observação dos dados de comprimento de fibras (Figura 1) evidencia uma tendência de valores crescentes ao longo da distância radial. Para a madeira de ambas as proveniências não houve diferenças estatisticamente significativas para os valores médios do comprimento das fibras entre as posições $100 \%$, 75\% e 50\%, sendo que apenas esta última (50\%) igualou-se estaticamente à posição $25 \%$, e a posição $0 \%$ diferiu de todas as outras.

O comprimento médio de fibras registrado na posição 100\% de ambos os plantios encontra-se dentro do limite da amplitude mencionada por Souza et al. (2003), os quais destacam, para a mesma espécie em análise, fibras com tamanho entre 1,10 e 1,59 mm o que as tornariam, segundo os mesmos autores, excelentes para fabricação de polpa e papel de fibra curta.

Observa-se ainda que para ambas as proveniências o teste de médias para o comprimento de fibras em função da posição radial revelou estabilização dos valores a partir da posição a $75 \%$ do raio. Urbinati (2013), em estudo com a madeira de Schizolobium amazonicum aos 14 anos, encontrou que na direção radial o comprimento de fibras aumentou de forma significativa até $13,5 \mathrm{~cm}$, 
quando então tendeu a estabilizar, sendo tal posição muito próxima à distância correspondente a $75 \%$ do raio $(13,8 \mathrm{~cm})$, adotada no presente trabalho. Essa mesma autora, levando em consideração o comprimento e a espessura da parede de fibras, definiu como limite para o lenho juvenil a posição condizente com a distância radial de $10,5 \mathrm{~cm}$, cujo valor, no presente estudo, se encontra entre as posições $50 \%$ e $75 \%$.
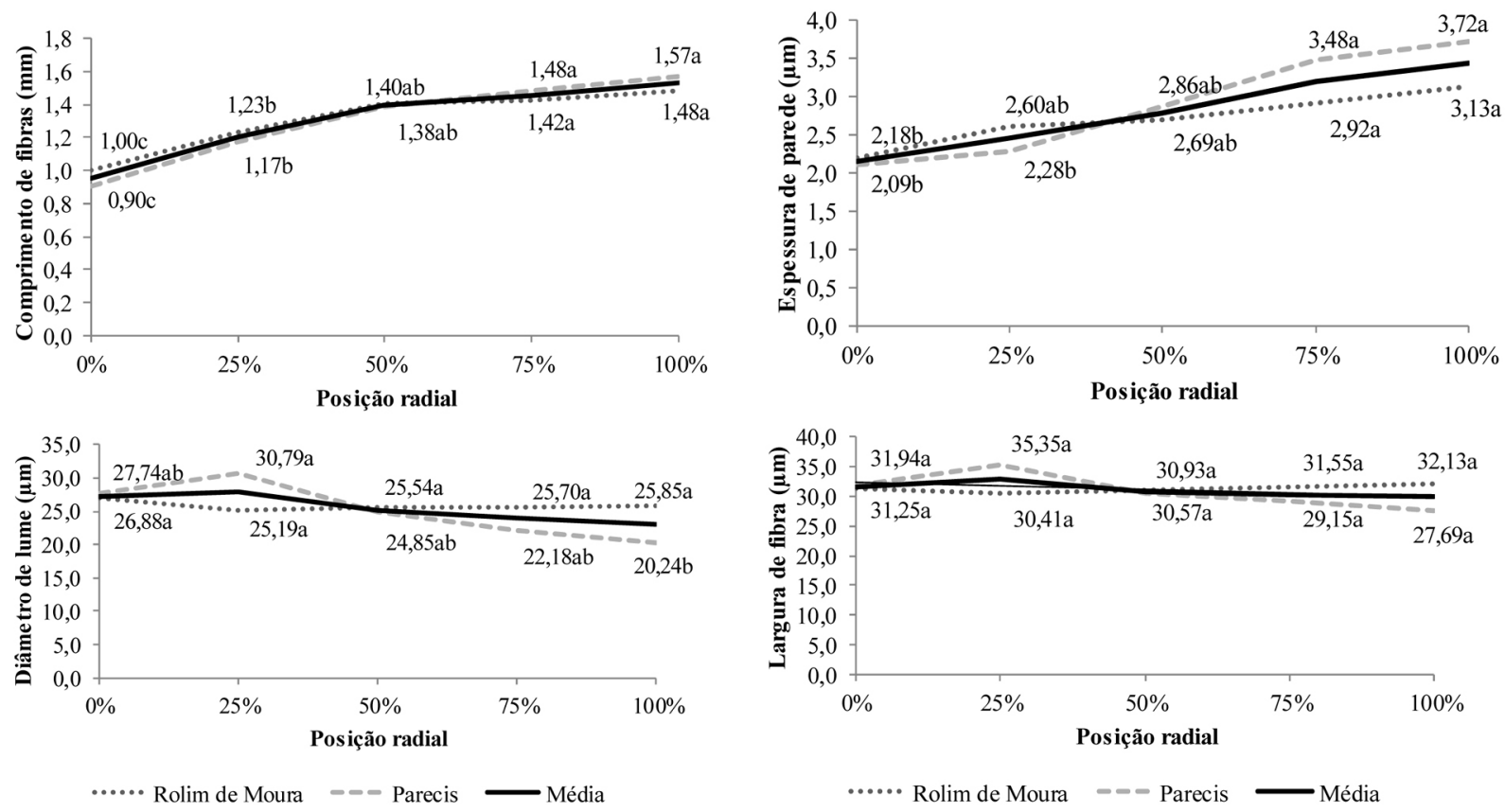

Figura 1. Variação radial do comprimento $(\mathrm{mm})$, espessura $(\mu \mathrm{m})$, largura de fibras $(\mu \mathrm{m})$ e diâmetro de lume ( $\mu \mathrm{m})$ de fibras para a madeira de Schizolobium amazonicum aos 14 anos.

Figure 1. Radial variation of length $(\mathrm{mm})$; thickness $(\mu \mathrm{m})$; fiber width $(\mu \mathrm{m})$ and lumen diameter $(\mu \mathrm{m})$ of fibers for Schizolobium amazonicum wood at age 14.

Com relação à espessura de parede, observa-se que embora os valores tenham mostrado um comportamento de elevação ao longo da distância radial, a porcentagem de incremento não se mostrou regular.

A amplitude observada para a espessura da parede de fibras entre as posições $0 \%$ e 100\% para cada proveniência avaliada e média geral é condizente com o intervalo mencionado por Urbinati (2013), 2 a 4 m. Da mesma forma, a amplitude do diâmetro de lume, entre as mesmas posições (0\% e 100\%), foi próxima também à observada pela referida autora (21 a $27 \mu \mathrm{m})$. Observa-se que os valores de largura de fibra e diâmetro de vaso dos extremos são muito próximos também aos verificados por Lobão et al. (2012), com valores de 32,48 $\mu \mathrm{m}$ e 27,62 $\mu \mathrm{m}$ para a largura de fibras e diâmetro de lume na posição mais próximo à medula e valores de 29,35 $\mu \mathrm{m}$ e 22,57 $\mu \mathrm{m}$ para estes mesmos parâmetros na posição próxima a casca. A variação radial do diâmetro de lume e largura de fibra seguiu o mesmo comportamento registrado para a espessura de parede.

O comportamento radial médio da madeira dos plantios avaliados revelou um aumento no valor de largura de fibra e diâmetro de lume entre as posições $0 \%$ e $25 \%$ seguido de um decréscimo no valor das médias, de forma análoga ao observado por Lobão et al. (2012) a partir de árvores de DAP médio de 27,4 cm de S. amazonicum, cuja tendência de elevação das médias foi registrada entre a posição $0 \%$ e a região de formação do cerne. Urbinati (2013) também registrou o mesmo comportamento para a largura de fibras, em que houve um aumento entre os valores das duas primeiras posições avaliadas, seguida de uma redução gradual nas posições seguintes.

\section{Coeficientes indicativos de qualidade da madeira}

Na Tabela 2 se encontra a estatística descritiva para a média dos coeficientes indicativos de qualidade da madeira de S. amazonicum para produção de polpa e papel. Os mesmos foram calculados segundo metodologia descrita por Foelkel e Barrichelo (1975). 
Modes et al. - Caracterização morfológica e química da madeira de

Schizolobium amazonicum aos 14 anos de plantios no estado de Rondônia

Tabela 2. Coeficientes indicativos de qualidade da fibra de Schizolobium amazonicum aos 14 anos em função da posição radial

Table 2. Indicative coefficients of Schizolobium amazonicum fiber quality at 14 years according to radial position.

\begin{tabular}{|c|c|c|c|c|c|c|c|c|}
\hline \multirow{2}{*}{ Indicativos } & \multicolumn{5}{|c|}{ Posição radial } & \multirow{2}{*}{ Média } & \multirow{2}{*}{ Desvio Padrão } & \multirow{2}{*}{ CV (\%) } \\
\hline & $0 \%$ & $25 \%$ & $50 \%$ & $75 \%$ & $100 \%$ & & & \\
\hline FP (\%) & 13,81 & 15,44 & 18,61 & 21,80 & 23,89 & 18,71 & 6,00 & 32,07 \\
\hline CF (\%) & 86,19 & 84,56 & 81,39 & 78,20 & 76,11 & 81,29 & 6,00 & 7,38 \\
\hline IR & 0,16 & 0,19 & 0,23 & 0,29 & 0,33 & 0,24 & 0,10 & 43,52 \\
\hline
\end{tabular}

Em que: FP: Fração parede; CF: Coeficiente de flexibilidade; IR: índice de Runkel

Analisando os dados da Tabela 2, verifica-se que o índice de Runkel calculado, menor que 0,25 permite enquadrar a madeira de Schizolobium amazonicum aos 14 anos no grupo I (VASCONCELOS, 2005). Esse resultado é importante, pois, madeiras pertencentes ao referido grupo são de excelente qualidade para a fabricação de papel. Dessa forma, em comparação com espécies de maior índice de Runkel, as fibras da madeira da espécie em referência estão sujeitas a um maior grau de colapso, permitindo assim a formação de uma maior superfície de contato entre fibras, resultando na produção de um papel de maior resistência à tração e ao estouro. O elevado valor médio do coeficiente de flexibilidade $(81,29 \%)$ confirma a maior susceptibilidade das fibras ao achatamento na produção de papel, com ganhos em resistência para o produto manufaturado.

A qualidade das fibras de S. amazonicum para a finalidade de produção de polpa e papel é reforçada pelo valor do índice fração parede calculado, inferior a 40\%, uma vez que Foelkel e Barrichelo (1975) mencionam que porcentagens acima desse valor não produzem celulose de boa qualidade porque as fibras são extremamente rígidas e pouco flexíveis.

Ao comparar os coeficientes indicativos de qualidade das fibras de S. amazonicum aos 14 anos com a madeira do gênero Eucalyptus, de reconhecido uso na produção de polpa e papel, comprova-se o potencial de suas fibras para o referido destino. Ferreira et al. (2006), estudando a madeira de 12 clones de Eucalyptus em idade de corte ( 9 anos), observaram uma fração parede de 46,66\% e um coeficiente de flexibilidade de 53,67\%, ou seja, diferente do observado no presente estudo, uma fração parede superior (até mesmo à porcentagem limite de 40\%) e um coeficiente de flexibilidade inferior.

\section{Morfologia de vasos da madeira de Schizolobium amazonicum}

A análise fatorial acusou influência do fator proveniência nas médias de diâmetro de vaso e área ocupada pelos vasos, com as maiores médias registradas para a madeira proveniente de Rolim de Moura (Tabela 3).

Tabela 3. Teste de médias para as variáveis dos elementos de vaso de S. amazonicum aos 14 anos, em função da proveniência.

Table 3. Means test for the variables of the vessels elements of S. amazonicum at 14 years, by origin.

\begin{tabular}{lccc}
\hline \multirow{2}{*}{ Proveniência } & \multicolumn{3}{c}{ Variáveis dos Elementos de Vaso } \\
\cline { 2 - 4 } & Diâmetro $(\boldsymbol{\mu m})$ & Frequência $\left(\mathbf{v a s o s} / \mathbf{m m}^{2}\right)$ & Área ocupada $(\%)$ \\
\hline Parecis & $186,93 \mathrm{~b}^{*}$ & $1,71 \mathrm{a}$ & $4,91 \mathrm{~b}$ \\
Rolim de Moura & $201,21 \mathrm{a}$ & $1,94 \mathrm{a}$ & $5,65 \mathrm{a}$ \\
Média & 194,07 & 1,82 & 5,28 \\
CV $(\%)$ & 10 & 23 & 19 \\
\hline
\end{tabular}

Em que: * Médias seguidas pela mesma letra não diferem estatisticamente entre si pelo teste de Tukey $(p \geq 0,05) ; C V=$ Coeficiente de variação

Rigatto et al. (2004) estudando a influência dos atributos de solo sobre a qualidade da madeira, relataram que a textura do solo influenciou a morfologia dos traqueídeos, sendo maior o diâmetro de lume observado na madeira formada em sítios de textura argilosa, em relação ao da madeira formada em sítios com solo de textura média.

Levando em consideração o percentual de argila registrada no solo dos plantios e os limites da Escala de Atterberg para a classificação da textura do mesmo pode-se classificar o solo do plantio estabelecido no município de Parecis $(27,57 \%)$ como de textura média (teor de argila entre $15 \%$ a $35 \%)$ e o solo do plantio estabelecido no município de Rolim de Moura $(39,16 \%)$ como de textura argilosa (teor de argila entre 35\% a 60\%). 
Assim o comportamento dos parâmetros de elementos de vaso registrado para a madeira dos plantios corroboram a informação de Rigatto et al. (2004), uma vez que os valores médios por posição radial referente ao diâmetro, frequência e área ocupada pelos vasos (Figura 2) na madeira extraída do plantio de textura argilosa (Rolim de Moura), apresentaram tendência de se manter sempre superiores ao longo de toda a distância radial, em relação aos valores médios da madeira proveniente da área com solo de textura média (Parecis), resultando em média superior (Tabela 3).

Portanto, essa característica física do solo do plantio no município de Rolim de Moura, provavelmente, condicionou uma maior disponibilidade hídrica no solo, e como uma adaptação dos indivíduos em crescimento nessas condições, houve um acréscimo do diâmetro dos vasos condutores e, por consequência, da área ocupada pelos mesmos. Roque et al. (2007) observaram que as alterações na anatomia do lenho, com respeito às células importantes para o fluxo de seiva mineral (vasos) e reserva de nutrientes (parênquima), foram induzidas pelas condições ecológicas dos locais de estudo, indicando que estes são os elementos anatômicos que refletem as mudanças do ambiente.

A média de diâmetro de vasos dos plantios avaliados no presente estudo é próxima a registrada no estudo Vidaurre (2010) em povoamentos com idades de 7 e 9 anos.

A frequência de vasos e a área ocupada pelos vasos foram compatíveis com o registrado no estudo de Lobão et al. (2012), com indivíduos em cuja amplitude de idade se encaixa a madeira avaliada (14 anos).

Na Figura 2 está representado o comportamento radial médio e de cada plantio avaliado, dos parâmetros de elementos de vaso (diâmetro, frequência e área ocupada).
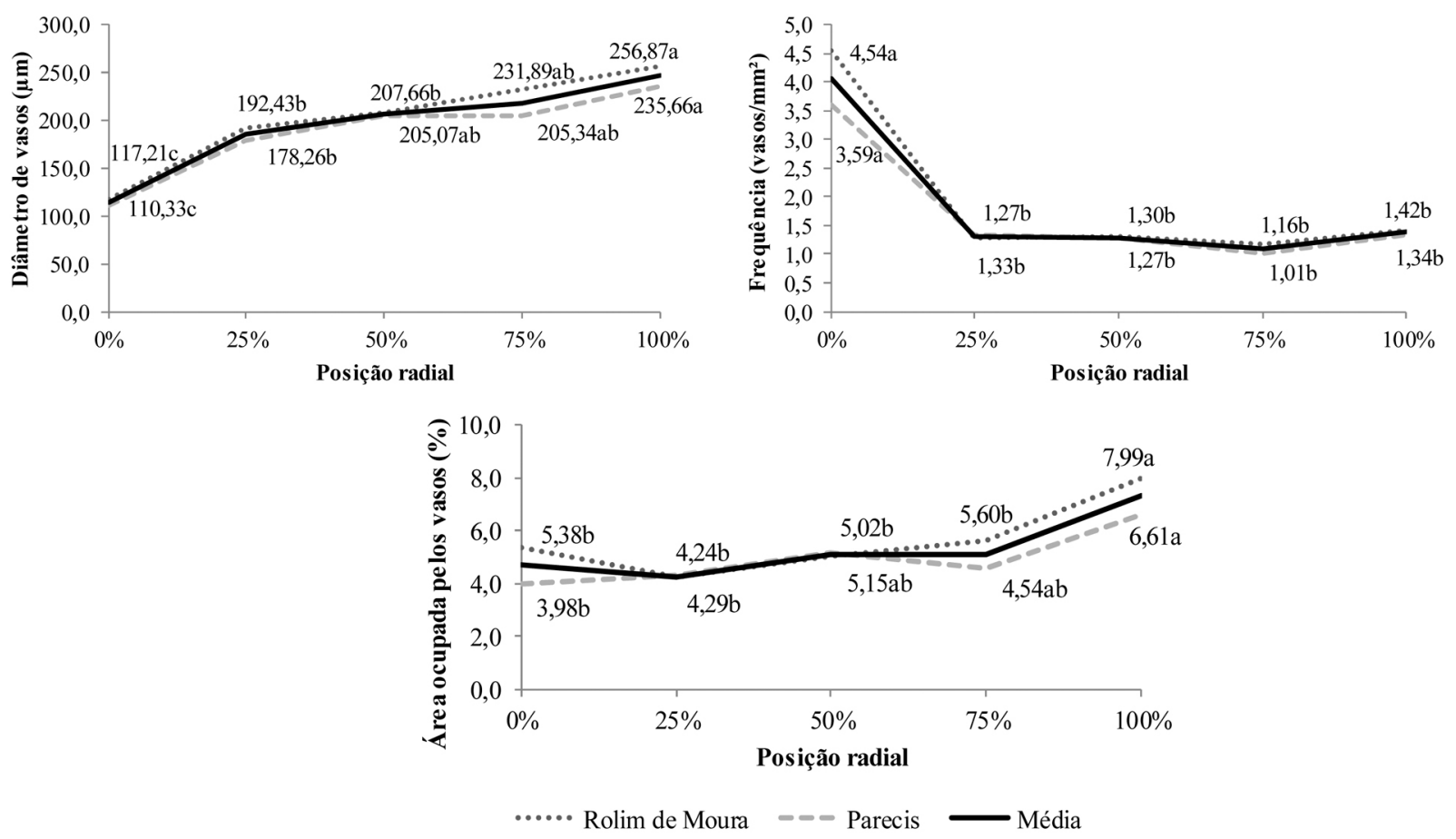

Figura 2. Variação radial do diâmetro de vasos $(\mu \mathrm{m})$, frequência (vasos $/ \mathrm{mm}^{2}$ ) e área ocupada pelos vasos $(\%)$ para a madeira de $S$. amazonicum aos 14 anos.

Figure 2. Radial variation of vessel diameter $(\mu \mathrm{m})$, frequency (vessels $/ \mathrm{mm}^{2}$ ) and area occupied by vessels $(\%)$ for S. amazonicum wood at age 14 .

Com relação aos valores médios de diâmetro dos vasos, observam-se valores crescentes ao longo da distância radial para a madeira de ambas as proveniências avaliadas. Com relação ao comportamento médio dos plantios avaliados no que se refere ao diâmetro de vaso, observa-se diferenciação estatística entre as médias das posições $0 \%$ e 100\%, com a média da posição 50\% sendo estatisticamente igual à das posições $25 \%$ e $75 \%$.

Vidaurre (2010) relatou para o S. amazonicum uma baixa frequência de elementos de vaso por milímetro quadrado, em média de 3 a 4, em que os maiores valores foram encontrados na região da madeira juvenil, entre $1,5 \mathrm{~cm}$ e $4,5 \mathrm{~cm}$ da medula, e os menores valores, próximos à casca (1/ $\mathrm{mm}^{2}$ ), portanto, condizentes aos encontrados no presente estudo. De acordo com Sette Júnior et al. 
(2012), sob o aspecto fisiológico, as alterações no diâmetro, frequência e disposição dos elementos de vasos são decorrentes da necessidade das plantas aumentarem sua capacidade de transporte de água e sais minerais, à medida que se processa o seu crescimento, resultando em aumento das dimensões de tais elementos. Vidaurre (2010) observou correlação positiva entre a idade de indivíduos de $S$. amazonicum e diâmetro de vasos, fato que deve ser levado em consideração nos processos de industrialização da madeira, uma vez que para a referida autora o $S$. amazonicum aparenta ser uma espécie com facilidade para penetração e passagem de fluidos, ou seja, trata-se de madeira permeável.

Quanto à frequência de vasos, para a madeira de ambas as proveniências, o valor médio encontrado próximo a medula (posição $0 \%$ do raio) foi superior e diferiu estatisticamente de todas as demais posições seguintes, as quais equivaleram-se entre si, mesma tendência também verificada para a média dos dois plantios por posição. Comportamento semelhante foi registrado por Urbinati (2013), cujo trabalho os maiores valores da frequência de vasos da madeira de S. amazonicum foram encontrados nos primeiros centímetros da medula, tendendo a entrar em estabilidade nas posições posteriores.

O comportamento inverso dos valores médios do diâmetro de vasos em relação a frequência (vasos $/ \mathrm{mm}^{2}$ ) ao longo do comprimento radial, também foi relatado por Vidaurre (2010), Lobão et al. (2012) e Urbinati (2013), estudando a mesma espécie em referência.

Com relação à área ocupada pelos vasos, em \%, observa-se que para ambas as proveniências houve aumento dessa variável ao longo da distância radial, com oscilação das médias entre as posições $25 \%$, 50\% e $75 \%$.

\section{Composição química da madeira de Schizolobium amazonicum}

Na Figura 3 consta a composição química das madeiras de Schizolobium amazonicum aos 14 anos, provenientes de Parecis - RO e Rolim de Moura - RO.

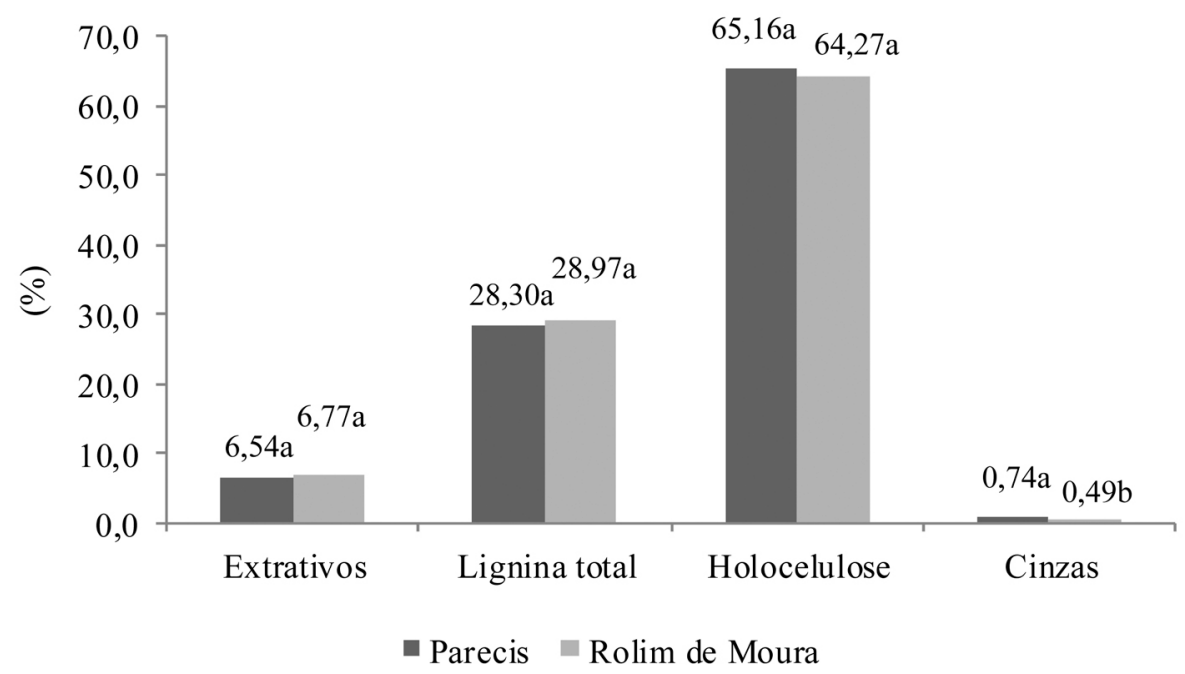

Figura 3. Valores médios dos componentes químicos da madeira de Schizolobium amazonicum Huber ex Ducke aos 14 anos, provenientes de Parecis - RO e Rolim de Moura - RO.

Figure 3. Mean values of the chemical components of Schizolobium amazonicum Huber ex Ducke wood at age 14 from Parecis - RO and Rolim de Moura - RO.

Observa-se que houve diferença estatisticamente significativa entre as proveniências apenas para o teor de cinzas. Segundo Oliveira (2003) o teor, assim como a composição química das cinzas podendo incluir cálcio, potássio, magnésio e traços de outros, pode ser afetado pela disponibilidade desses minerais no solo.

A correlação de Pearson realizada (Tabela 4) permitiu verificar que a concentração de cinzas, em porcentagem na madeira de S. amazonicum, esteve condicionada de forma inversa com os atributos que restringem a disponibilidade de nutrientes no solo tais como aqueles ligados à acidez do solo, como a quantidade de alumínio trocável (Al), a saturação por alumínio (m\%) e a concentração de ferro (Fe) no solo. Segundo Carvalho et al. (2005) com relação ao ferro, classificado como mi- 
cronutriente em virtude da menor necessidade pelas plantas em quantidade, quando em maior concentração no solo tem sua absorção restrita a fim de evitar toxidez pela planta, em benefícios de outros nutrientes.

Tabela 4. Matriz de correlação significativa entre as variáveis anatômicas e químicas da madeira de S. amazonicum e as propriedades do solo.

Table 4. Matrix of significant correlation between the anatomical and chemical variables of S. amazonicum wood and soil properties.

\begin{tabular}{|c|c|c|}
\hline Correlação & Coef. Correlação (r) & Signif. \\
\hline Holocelulose (\%) x Argila (\%) & $-0,8275$ & * \\
\hline Holocelulose (\%) x P (mg. $\left.\mathrm{dm}^{3}\right)$ & 0,8113 & * \\
\hline Holocelulose (\%) x Ca (mmolc. $\left.\mathrm{dm}^{3}\right)$ & 0,7329 & * \\
\hline Holocelulose (\%) x Mg (mmolc. $\left.\mathrm{dm}^{3}\right)$ & 0,7286 & * \\
\hline Holocelulose (\%) x Al (mmolc. $\left.\mathrm{dm}^{3}\right)$ & $-0,7609$ & * \\
\hline Holocelulose $\times \mathrm{H}+\mathrm{AL}\left(\mathrm{mmolc} . \mathrm{dm}^{3}\right)$ & $-0,7362$ & * \\
\hline Holocelulose (\%) x SB (mmolc. $\left.\mathrm{dm}^{3}\right)$ & 0,7388 & * \\
\hline Holocelulose (\%) x m (\%) & $-0,7471$ & * \\
\hline Cinzas $(\%) \times$ Densidade de solo $\left(\mathrm{g} / \mathrm{cm}^{3}\right)$ & 0,8915 & ** \\
\hline Cinzas $(\%)$ x Densidade de partículas $\left(\mathrm{g} / \mathrm{cm}^{3}\right)$ & 0,8531 & ** \\
\hline Cinzas (\%) x Macroporosidade (\%) & $-0,8802$ & ** \\
\hline Cinzas (\%) x Porosidade total (\%) & $-0,8801$ & ** \\
\hline Cinzas $(\%)$ x Cu $\left(\mathrm{mg} / \mathrm{dm}^{3}\right)$ & 0,7235 & * \\
\hline Cinzas $(\%) \times \mathrm{Fe}\left(\mathrm{mg} / \mathrm{dm}^{3}\right)$ & $-0,7646$ & * \\
\hline Cinzas $(\%) \times \mathrm{Mn}\left(\mathrm{mg} / \mathrm{dm}^{3}\right)$ & 0,7985 & * \\
\hline Cinzas $(\%) \times \mathrm{K}\left(\mathrm{mg} / \mathrm{dm}^{3}\right)$ & 0,8064 & * \\
\hline Cinzas (\%) x Al (mmolc. $\left.\mathrm{dm}^{3}\right)$ & $-0,7333$ & * \\
\hline Cinzas (\%) x V (\%) & 0,7313 & * \\
\hline Cinzas (\%) x m (\%) & $-0,7602$ & * \\
\hline
\end{tabular}

Em que: ** significativo ao nível de $1 \%$ de probabilidade $(\mathrm{p}<.0 \mathrm{l})$; * significativo ao nível de $5 \%$ de probabilidade $(.0 \mathrm{O} \leq \mathrm{P}<.05) ; \mathrm{P}=$ fósforo; $\mathrm{Ca}=$ cálcio; $\mathrm{Mg}=$ magnésio; $\mathrm{Al}=$ alumínio; $\mathrm{H}+\mathrm{Al}=$ acidez potencial; $\mathrm{SB}=$ soma de bases trocáveis; $\mathrm{m}=$ saturação por alumínio; $\mathrm{Cu}=$ cobre; $\mathrm{Fe}=$ ferro; $\mathrm{Mn}=$ manganês; $\mathrm{K}=$ potássio; $\mathrm{Al}=$ alumínio; $\mathrm{V}=$ saturação da $\mathrm{CTC}$ por bases.

Os atributos químicos do solo dos plantios amostrados (Tabela 5) confirma o comportamento das correlações observadas, visto que o solo do plantio no município de Rolim de Moura, que apresentou menor porcentagem de cinzas em sua madeira (Figura 3), é um solo caracterizado como de baixos teores de $\mathrm{Ca}^{2+}, \mathrm{Mg}^{2+} e^{+}$, elevada quantidade de alumínio trocável $\left(\geq 3\right.$ mmolc $\left.\mathrm{dm}^{-3}\right)$ e saturação em alumínio trocável superior a 50\%, levando em consideração as tolerâncias estipuladas por Ronquim (2010).

Tabela 5. Atributos químicos do solo dos plantios amostrados.

Table 5. Chemical characterization of the soil of the sampled plantations.

\begin{tabular}{|c|c|c|c|c|c|c|c|c|c|c|}
\hline \multirow{2}{*}{ Proc. } & \multirow{2}{*}{$\begin{array}{c}\text { Prof. } \\
\mathbf{c m}\end{array}$} & \multirow{2}{*}{$\mathrm{MO} \mathrm{g} / \mathrm{dm}^{3}$} & \multirow{2}{*}{$\mathrm{pH}$} & $\mathbf{P}$ & $\mathrm{K}$ & $\mathrm{Ca}$ & $\mathrm{Mg}$ & Al & $\mathrm{H}+\mathrm{Al}$ & SB \\
\hline & & & & $\mathrm{mg}^{\mathrm{dm} \mathrm{m}^{-3}}$ & \multicolumn{6}{|c|}{ mmolc. $\mathrm{dm}^{-3}$} \\
\hline \multirow{2}{*}{$P$} & $0-10$ & 36 & 6,2 & 16 & 1,9 & 90 & 10 & $<1$ & 22 & 102,7 \\
\hline & $10-30$ & 26 & 6,0 & 8 & 2,0 & 50 & 7 & 0 & 24 & 59 \\
\hline \multirow{2}{*}{ RM } & $0-10$ & 32 & 3,6 & 6 & 1,2 & 3 & 2 & 14 & 125 & 6,2 \\
\hline & $10-30$ & 24 & 3,6 & 5 & 0,6 & 2 & $<1$ & 10 & 98 & 3,4 \\
\hline \multirow{2}{*}{ Proc. } & Prof. & CTC & $\mathbf{V}$ & $\mathbf{m}$ & B & $\mathrm{Cu}$ & $\mathrm{Fe}$ & Mn & $\mathrm{Zn}$ & \\
\hline & $\mathbf{c m}$ & mmolc. $\mathrm{dm}^{-3}$ & \multicolumn{2}{|r|}{$\%$} & \multicolumn{5}{|c|}{$\mathrm{mg} \mathrm{dm}^{-3}$} & \\
\hline \multirow{2}{*}{$P$} & $0-10$ & 124,7 & 81 & $<1$ & 0,16 & 4,5 & 40 & 121,4 & 21,7 & \\
\hline & $10-30$ & 83,1 & 70 & 0 & $<1$ & 6,0 & 36 & 121,4 & 29,1 & \\
\hline \multirow{2}{*}{ RM } & $0-10$ & 131,7 & 5 & 70 & 0,14 & 0,6 & 320 & 12,3 & 19,9 & \\
\hline & $10-30$ & 101,4 & 3 & 75 & 0,13 & 0,5 & 194,5 & 5,3 & 12,1 & \\
\hline
\end{tabular}

Em que: $\mathrm{P}=$ Parecis; $\mathrm{RM}=$ Rolim de Moura; $<\mathrm{I}=$ Menor que o limite de quantificação; $M . \mathrm{O}=$ matéria orgânica; $\mathrm{P}=$ fósforo; $\mathrm{K}=$ potássio; $\mathrm{Ca}=$ cálcio; $\mathrm{Mg}=$ magnésio; $\mathrm{Al}=$ alumínio; $\mathrm{H}+\mathrm{Al}=$ acidez potencial; $\mathrm{SB}=$ soma de bases trocáveis; $\mathrm{CTC}=\mathrm{Capacidade}$ de troca catiônica em $\mathrm{pH}$

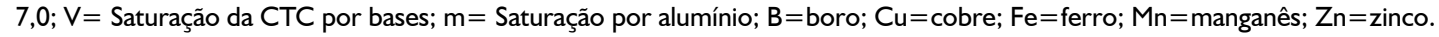

Observa-se que as propriedades físicas de densidade do solo e densidade de partículas correlacionaram positivamente em nível de $1 \%$ de probabilidade com o conteúdo de cinzas na madeira (Tabela 4). Segundo Silva et al. (2002) a absorção de nutrientes pelas plantas depende do crescimento 
e eficiência das raízes e da disponibilidade de nutrientes no solo. Desta forma, embora a densidade do solo ofereça uma maior restrição das raízes à exploração dos nutrientes, observa-se que o solo proveniente de Rolim de Moura, embora tenha sido caracterizado como de menor densidade, por ser considerado um solo muito pobre, conforme discussão relativa à Tabela 5, pode ter contribuído para redução da disponibilidade e acúmulo de nutrientes.

Ao comparar o valor médio da porcentagem de extrativos (6,65\%) (Figura 3) com os disponíveis na literatura para a madeira de $S$. amazonicum, observa-se que a porcentagem é mais próxima do observado por Vidaurre (2010) com a madeira de S. amazonicum aos 7 anos e superior em 20,30\% ao observado por Silva et al. (2013) para a madeira desta espécie em idade superior (15 anos).

O teor de extrativos, ora verificado na madeira estudada, é característica indesejável para madeiras destinadas à produção de celulose. Segundo Carvalho (1997), o aumento de extrativos na madeira, além de exigir maior carga de reagentes, também aumenta o potencial de formação de depósitos de "pitch" em equipamentos, principalmente telas e feltros, bem como afetam o potencial de absorção de líquidos do papel.

O valor médio do teor de lignina total da madeira $(28,63 \%)$ foi mais próximo ao observado por Almeida (2009) (28,8\%) que não fez referência à idade da madeira avaliada. Com relação à madeira de idade mais próxima (15 anos), foi superior aos 25,7\% observado por Silva et al. (2013).

Gomide et al. (2010), a partir de 75 amostras de madeiras de Eucalyptus spp. em idade comercial de corte, determinaram teor de extrativos médio de 3,08\% e teor de lignina total médio de 27,30\%, inferiores aos registrados para a madeira de $S$. amazonicum. Também a porcentagem registrada para os extrativos totais no presente estudo foi superior ao registrado por Lima et al. (2007) (5,54\%) para a madeira de E. urophylla de mesma idade (14 anos).

De acordo com Vidaurre (2010) apesar da madeira de S. amazonicum apresentar alto teor de lignina, a mesma pode ser facilmente deslignificada, já que Vidaurre (2010) ao avaliar a relação S/G na madeira desta espécie observou que a frequência das estruturas siringila (S) foi ligeiramente superior às estruturas guaiacila (G), que segundo Ferreira et al. (2006) são de mais fácil degradação pelos reagentes de cozimento.

O teor de holocelulose médio encontrado no presente estudo $(64,71 \%)$ está próximo ao encontrado por Vidaurre (2010), com um valor inferior em 6,63\% ao observado para a madeira de 15 anos estimada por Silva et al. (2013). Vidaurre (2010), estudando a composição química da madeira de S. amazonicum em diferentes idades, observou um aumento do teor de celulose na madeira com o aumento da idade, devido ao espessamento da parede celular, acompanhado de redução no teor de hemicelulose.

Considerando-se conjuntamente os dados obtidos para a madeira de ambos os plantios, o valor médio do teor de cinzas foi de 0,61\%. Esse valor é compatível com o encontrado por Melo et al. (2013) em idades entre 5 e 7 anos e Vidaurre (2010) na idade de 11 anos. O alto teor de cinzas registrado na madeira de S. amazonicum proveniente de Parecis (Figura 3) é uma característica indesejável na utilização dessa matéria prima na indústria de celulose e papel, principalmente em fábricas de celulose de ciclo de água muito fechado, com altas taxas de recirculações e baixos volumes de efluentes. Segundo Freddo et al. (1999) sendo os minerais uma fonte de contaminação dos efluentes, estes se acumulam no processo, causando problemas como corrosões, incrustações e entupimentos e, portanto, reduzem a vida útil dos materiais.

\section{CONCLUSÕES}

Os parâmetros referentes às fibras da madeira de S. amazonicum não foram influenciados pela proveniência;

Os valores do comprimento e espessura da parede de fibras na direção radial foram crescentes e tenderam a uma estabilização a partir da posição a $75 \%$ do raio, na madeira de ambas as proveniências avaliadas, o que pode ser um indicativo do início da formação de madeira adulta desta posição adiante;

O diâmetro de vasos e a sua porcentagem de área ocupada mostraram tendência de elevação dos valores na direção medula-casca, acompanhada de uma redução na frequência dos mesmos;

O diâmetro de vasos e área ocupada pelos mesmos foi o caractere anatômico que diferiu na madeira dos plantios amostrados. 
Com relação aos parâmetros químicos apenas o teor de cinzas diferiu entre as proveniências avaliadas, com porcentagem superior registrada para a madeira proveniente de Parecis;

Embora os coeficientes indicativos de qualidade das fibras da madeira de S. amazonicum aos 14 anos, tenham revelado grande aptidão desta espécie para produção de polpa e papel na idade considerada, recomenda-se que o elevado teor de extrativos observado seja levado em consideração nos programas de melhoramento genético para adequação da madeira avaliada.

\section{REFERÊNCIAS BIBLIOGRÁFICAS}

ALMEIDA, V. C. Efeito da adição de carga e extensor nas propriedades do adesivo uréia-formaldeído e dos compensados de pinus e paricá. 2009. 86 p. Dissertação (Mestrado em Ciência Florestal) - Universidade Federal de Viçosa, Viçosa, 2009.

CARVALHO, H. G. Efeito da idade de corte da madeira e de variáveis de refino nas propriedades da celulose kraft branqueada de eucalipto. 1997. 121 p. Dissertação (Mestrado em Ciências Florestais) - Universidade Federal de Viçosa, Viçosa, 1997.

CARVALHO, J. C. R.; SOUSA, C. S.; SOUSA, C. S. Fertilizantes e fertilização. Cruz das Almas: Universidade Federal da Bahia, Departamento de Química do Solo, 2005. 159 p.

CASTELO, P. A. R.; MATOS, J. L. M.; DEDECEK, R. A.; LAVORANTI, O. J. Influência de diferentes sítios de crescimento sobre a qualidade da madeira de Pinus taeda. Floresta, Curitiba, v. 38, n. 3, p. 495-506, 2008.

FERREIRA, C. R.; FANTINI JUNIOR, M.; COLODETTE, J. L.; GOMIDE, J. L.; CARVALHO, A. M. M. L. Avaliação tecnológica de clones de eucalipto: parte 1 - qualidade da madeira para produção de celulose Kraft. Scientia Forestalis, Piracicaba, n. 70, p. 161-170, 2006.

FREDDO, A.; FOELKEL, C. E. B.; FRIZZO, S. M. B.; SILVA, M. C. M. Elementos minerais em madeiras de eucaliptos e acácia negra e sua influência na indústria de celulose kraft branqueada. Ciência Florestal, Santa Maria, v. 9, n. 1, p. 193-209, 1999.

FOELKEL, C. E. B.; BARRICHELO, L. E. G. Relações entre características da madeira e propriedades da celulose e papel. O Papel, São Paulo, v. 36, n. 9, p. 49-53, 1975.

GOMIDE, J. L.; FANTUZZI NETO, H.; REGAZZI, A. J. Análise de critérios de qualidade da madeira de eucalipto para produção de celulose kraft. Revista Árvore, Viçosa, v. 34, n. 2, p. 339-344, 2010.

IAWA - INTERNATIONAL ASSOCIATION OF WOOD ANATOMISTS. List of microscopic features for hardwood identification. IAWA Bulletin, Oakland, v. 10, n. 3, p. 219-332, 1989.

IWAKIRI, S.; MATOS, J. L. M.; PINTO, J. A.; VIANA, L C.; SOUZA, M. M.; TRIANOSKI, R.; ALMEIDA, V. C. Produção de painéis laminados unidirecionais - LVL com lâminas de Schizolobium amazonicum, Eucalyptus saligna e Pinus taeda. Cerne, Lavras, v. 16, n. 4, p. 557-563, 2010.

JESUS, R. C. S. Características anatômicas e físicas da madeira de paricá (Schizolobium amazonicum Huber ex. Ducke), plantado em diferentes espaçamentos e condições ambientais no município de aurora do Pará - Pa. 2004. 165 p. Dissertação (Mestrado em Ciências Florestais) - Universidade Federal Rural da Amazônia, Belém, 2004.

LIMA, C. K. P.; MORI, F. A.; MENDES, L. M.; CARNEIRO, A. C. O. Características anatômicas e química da madeira de clones de Eucalyptus e sua influência na colagem. Cerne, Lavras, v. 13, n. 2, p. 123-129, 2007. 
Modes et al. - Caracterização morfológica e química da madeira de

Schizolobium amazonicum aos 14 anos de plantios no estado de Rondônia

LOBÃO, M. S. Dendrocronologia, fenologia, atividade cambial e qualidade do lenho de árvore de Cedrela odorata L., Cedrela fissilisVell. E Schizolobium parahyba var. amazonicum Hub. ex Ducke, no estado do Acre, Brasil. 2011. 215 p. Tese (Doutorado em Recursos Florestais) - Escola Superior de Agricultura "Luiz de Queiroz", Universidade de São Paulo, 2011.

LOBÃO, M. S.; COSTA, D. P.; ALMONACID, M. A.; TOMAZELLO FILHO, M. Qualidade do lenho de árvores de Schizolobium parahyba var. amazonicum, Acre, Brasil. Floresta e Ambiente, Seropédica, v. 19, n. 3, p. 374$384,2012$.

MELO, R. R.; DEL MENEZZI, C. H. S.; SOUZA, M. R., STANGERLIN, D. M. Avaliação das propriedades físicas, químicas, mecânicas e de superfície de lâminas de Paricá (Schizolobium amazonicum Huber ex. Ducke). Floresta e Ambiente, Seropédica, v. 20, n. 2, p. 238-249, 2013.

OLIVEIRA, E. Características anatômicas, químicas e térmicas da madeira de três espécies de maior ocorrência no semi-árido nordestino. 2003. 122 p. Tese (Doutorado em Ciências Florestais) - Universidade Federal de Viçosa, Viçosa, MG, 2003.

RIBEIRO, V. C. N. Caracterização anatômica da matéria-prima e avaliação das propriedades tecnológicas de painéis MDF de madeira de Schizolobium amazonicum Huber ex Ducke e de resíduos de espécies da Amazônia. 2015. 124 p. Dissertação (Mestrado em Recursos Florestais) - Escola Superior de Agricultura "Luiz de Queiroz", Universidade de São Paulo, 2015.

RIGATTO, P. A.; DEDECEK, R. A.; MATOS, J. L. M. Influência dos atributos do solo sobre a qualidade da madeira de Pinus taeda para produção de celulose Kraft. Revista Árvore, Viçosa, v. 28, n. 2, p. 267-273, 2004.

RONQUIM, C. C. Conceitos de fertilidade do solo e manejo adequado para as regiões tropicais. Campinas: Embrapa Monitoramento por Satélite, 2010. 26 p. (Boletim de Pesquisa e Desenvolvimento, 8).

ROQUE, R. M.; TOMAZELLO FILHO, M.; DIAS, C. T. S. Variações na estrutura da anatomia do lenho de árvores de Gmelina arbórea Roxb. (Verbenaceae) de clima tropical seco e úmido na Costa Rica. Scientia Forestalis, Piracicaba, n. 75, p. 65-75, 2007.

SETTE JÚNIOR, C. R.; OLIVEIRA, I. R.; TOMAZELLO FILHO, M.; YAMAJI, F. M.; LACLAU, J. P. Efeito da idade e posição de amostragem na densidade e características anatômicas da madeira de Eucalyptus grandis. Revista Árvore, Viçosa, v. 36, n. 6, p. 1183-1190, 2012.

SILVA, J. J. N.; CARDOSO, G. V.; SILVA JÚNIOR, F. G.; STANGERLIN, D. M. Caracterização tecnológica da madeira de Schizolobium amazonicum para a produção de celulose Kraft. Ciência da Madeira, Pelotas, v. 4, n. 1, p. 33-45, 2013.

SILVA, M. G.; MORI, F. A.; FERREIRA, G. C.; RIBEIRO, A. O.; CARVALHO, A. G.;

BARBOSA, A. C. M. C. Estudo anatômico e físico da madeira de Schizolobium parahyba var. amazonicum proveniente de povoamentos nativos da Amazônia Oriental. Scientia Forestalis, Piracicaba, v. 44, n. 110, p. 293-301, 2016.

SILVA, S. R.; BARROS, N. F.; NOVAIS, R. F.; PEREIRA, P. R. G. Eficiência nutricional de potássio e crescimento de eucalipto influenciados pela compactação do solo. Revista Brasileira de Ciência do Solo, Viçosa, v. 26, n. 4, p. 1001-1010, 2002.

SOUZA, C. R.; ROSSI, L. M. B.; AZEVEDO, C. P. Paricá: Schizolobium parahyba var. amazonicum (Huber x Ducke) Barneby. Manaus: Embrapa Amazônia Ocidental, 2003. 12 p. (Circular Técnica, 18). 
TAPPI - TECHNICAL ASSOCIATION OF PULP AND PAPER INDUSTRY. Test Methods. Atlanta: TAPPI Press, 2007. $2 \mathrm{v}$.

TEREZO, R. F. Avaliação tecnológica do paricá e seu uso em estruturas de madeira laminada colada. 2010. 201 p. Dissertação (Doutorado em Engenharia Civil) - Universidade Federal de Santa Catarina, Florianópolis, 2010.

URBINATI, C. V. Influência das características anatômicas em juntas coladas de Schizolobium parahyba var. amazonicum (Huber ex. Ducke) Barneby (Paricá). 2013. 162 p. Tese (Doutorado em Ciência e Tecnologia da Madeira) - Universidade Federal de Lavras, Lavras, 2013.

VASCONCELOS, F. S. R. Avaliação do processo SuperBatch ${ }^{\mathrm{TM}}$ de polpação de Pinus taeda. 2005. 106 p. Dissertação (Mestrado em Recursos Florestais) - Escola Superior de Agricultura "Luiz de Queiroz", Universidade de São Paulo, Piracicaba, 2005.

VIDAURRE, G. B. Caracterização anatômica, física e químico-mecânica da madeira de paricá (Schizolobium amazonicum) para produção de energia e polpa celulósica. 2010. 74 p. Tese (Doutorado em Ciências Florestais) - Universidade Federal de Viçosa, Viçosa, 2010. 
